\title{
Pigmented and polypoid tumor of the pubis
}

\author{
Mariem Rekik ${ }^{1}$, Khadija Sellami ${ }^{2}$, Fatma Hammami ${ }^{1}$, Massara Baklouti ${ }^{1}$, and Hamida \\ Turki $^{1}$ \\ ${ }^{1}$ Hedi Chaker Hospital \\ ${ }^{2}$ Hopital Hedi Chaker
}

February 16, 2022

\begin{abstract}
Seborrheic keratosis is a common benign epidermal tumor occurring in patients aged over 50 years. It is located preferentially in the trunk, head and neck. The genital location is rare. We report a case of 59 year-old-men presenting a seborrheic keratosis of the pubis.

Introduction :

Seborrheic keratosis (SK) is a common benign epidermal tumor with predominance in adult patients aged over 50 years. It is preferentially located in the trunk, head and neck. The genital location is rare. We report an original case of 59 year-old-men presenting a SK of the pubis.
\end{abstract}

Case presentation :

A 59 year-old-men, with no medical history, presented to our department for a 15-year history of a gradually enlarged and pigmented tumor of the pubis. Physical examination showed humped, well defined, verrucous lesion measuring $6 \mathrm{~cm}$ in diameter with a rough and warty appearance (Fig.1 ). No ulceration, crusts, or bleeding were noted. Dermoscopy revealed cerebriform convolutions with densely packed exophytic papillary structures which were separated by black irregular crypts. Black comedo-like openings, fissures as well as hairpin and dotted vessels were present (Fig.2 ). The lesion was excised. Histological examination revealed papillomatous hyperplasia of the epidermis with hyperpigmentation of the basal membrane. It also showed tunnels and multiple pseudohorn cysts with ortho-keratotic hyperkeratosis. The dermis was richly inflammatory with no melanocytic cells. There were no koïlocytes. The diagnosis of SK was retained.

Discussion :

SK is a common benign epidermal tumor with predominance in adult patients aged over 50 years. They are preferentially located in the trunk, head and neck. The genital location is rare and can mimic genital warts (1). Different clinical and histologic subtypes have been identified (hyperkeratotic type, acanthotic type, reticular/adenoid type, clonal type, irritated type, regressing type, melanoacanthoma, and verrucous SK with keratoacanthoma-like features) (2). For our patient, the histologic features match more with the hyperkeratotic type in which hyperkeratosis and papillomatosis of the epidermis are pronounced (3).

The clinical variability of SK raises some diagnostic difficulties. Dermoscopy is the preferred non-invasive diagnostic method. Most cases of SK exhibit the typical dermoscopic findings of fissures and ridges, hairpin vessels with white halo, comedo-like openings, and milia-like cysts. Histopathologically, these dermoscopic characteristics correspond to papillomatous surface of the epidermis, enlarged capillaries of the dermal papillae, pseudohorn cysts in the epidermis opened to the surface of the lesion and intraepidermal cysts, respectively (3). 
Dermoscopy of genital warts reveals distinctive patterns and vascular features (4). The pattern, mosaic in flat lesion, fingerlike and knoblike in papillomatous lesions (5), reflects the life stages of genital warts. The vascular features include hairpin, glomerular or dotted vessels (4). Unna nevus is a variant of melanocytic nevus. It corresponds to a papillomatous dermal or compound nevus. Clinically, it presents as a soft polypoid or sessile lesion, usually papillomatous, light- to dark-brown in colour (6). On dermoscopy, it shows a typical globular pattern composed of numerous tan to dark-brown, round to oval globules and can in some instances display exophytic papillary structures (6). Moreover, dermoscopy usually shows, especially at the periphery, the typical "comma-like" vessels (6). Sometimes, milia like cysts and comedo-like openings are also detected by dermoscopy (3). The wobble sign is useful to distinguish Unna nevus from SK, as papillomatous dermal nevi, unlike SK, wobble when the dermoscope is pushed from site to site (6).

Conclusion :

Making a definitive diagnosis of SK can be challenging. The dermoscopy facilitates the diagnosis but histology may be necessary in some cases especially in pubic location. In the latter, dermoscopy may show papillary structures which can be seen in SK but also in unna nevus and genital warts.

Author contribution:

RM and SK: wrote the manuscript. AM: revised the manuscript. BM, HF, TH: contributed to the management of the patient and revised the article. TH: critically reviewed the manuscript and gave final approval. All authors have read and approved the final manuscript and agree to take full responsibility for the integrity and accuracy of the work.

References:

1. Naciri I, Ismaili N. Unusual location of giant seborrhoeic keratoses. Pan Afr Med J. 2017 Sep 25;28:72.

2. Wollina U. Recent advances in managing and understanding seborrheic keratosis. F1000Res. 2019 Aug $28 ; 8$.

3. Minagawa A. Dermoscopy-pathology relationship in seborrheic keratosis. J Dermatol. 2017 May;44(5):518524.

4. Dong H, Shu D, Campbell TM et al. Dermatoscopy of genital warts. J Am Acad Dermatol. 2011 May;64(5):859-64.

5. Ozdemir F, Kilinc-Karaarslan I, Akalin T. A pigmented, hemorrhagic genital wart: clinical, dermoscopic, and histopathologic features. Arch Dermatol. 2008 Aug;144(8):1072-3.

6. Soyer, H.P., Argenziano, G., Hofmann-Wellenhof, R., Johr, R.H. (2007). Color Atlas of Melanocytic Lesions of the Skin. Berlin. Springer, 333p 


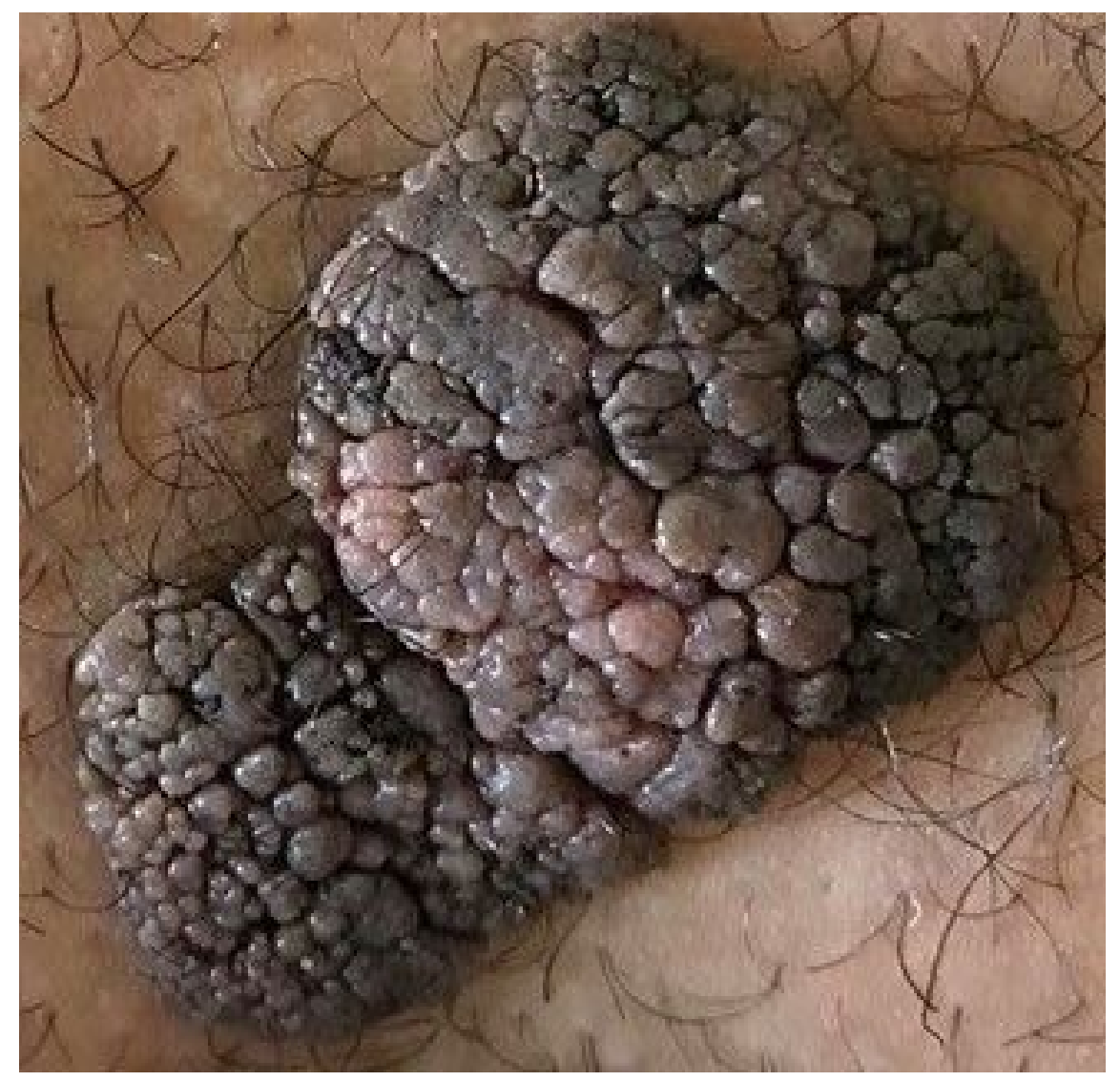




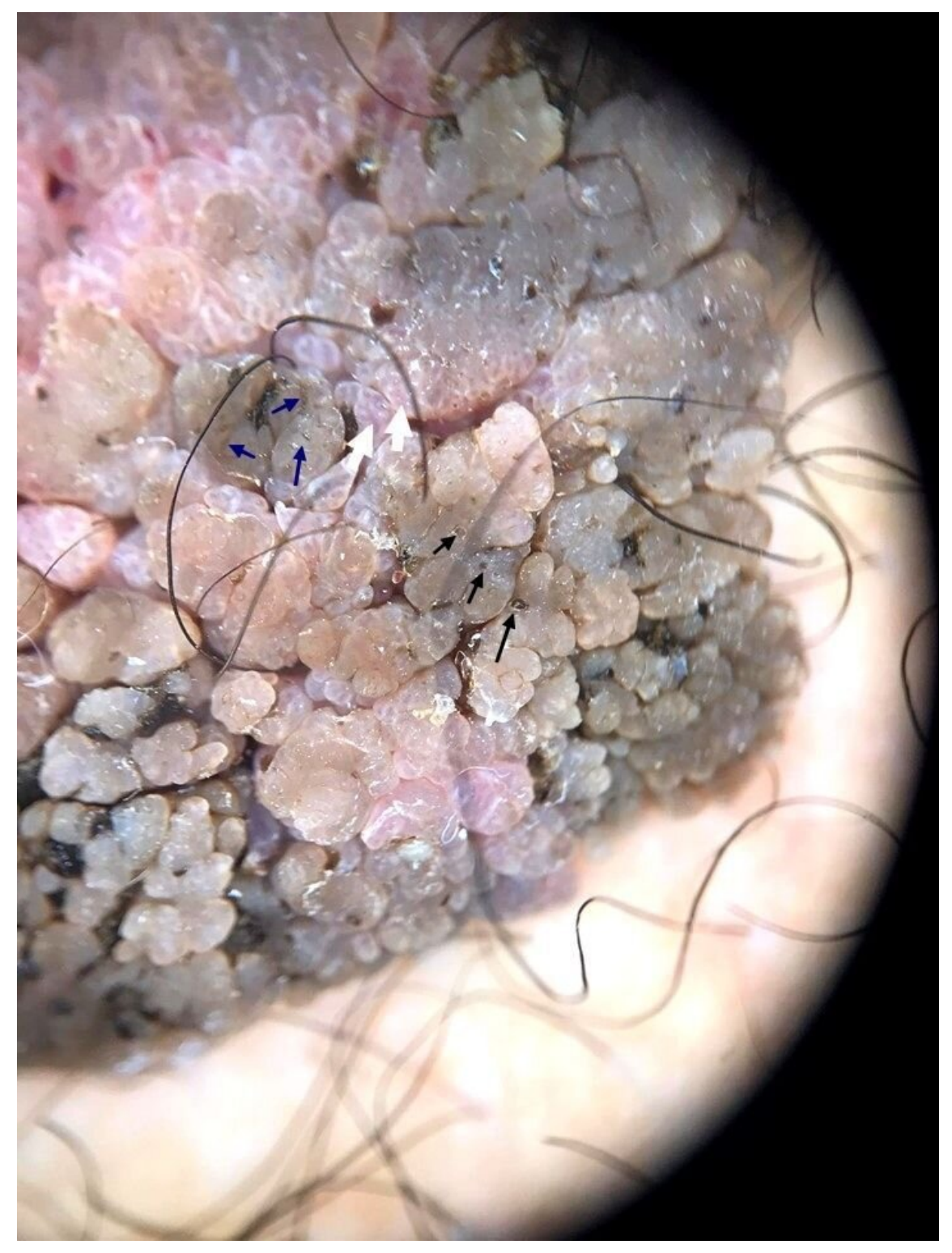

